\title{
Constitutional methylation of cancer-related and selenoprotein coding genes in breast carcinoma in Polish population
}

\author{
Satish Gupta ${ }^{1,3^{*}}$, Katarzyna Jaworska ${ }^{1,3}$, Anna Jakubowska ${ }^{1}$, Tomasz K Wojdacz ${ }^{2}$, Lise Lotte Hansen², Jan Lubiński ${ }^{1}$ \\ From Annual Conference on Hereditary Cancers 2012 \\ Szczecin, Poland. 30-31 August 2012
}

Recently, much attention is paid to the phenomenon of gene's hypermethylation in the peripheral blood (PB) and its involvement in the pathology of cancer. Breast cancer is a complex disease driven by multiple factors including both genetic and epigenetic processes. Genetic changes associated with breast cancer are not completely known, but epigenetic mechanisms involved in this disease seem to play an important role in its pathophysiology. An aberrant methylation in the promoter regions of genes involved in cancer induction and promotion, like BRCA1, BRCA2, ATM, MLH1 and ESR1, may be of particular importance in breast cancer.

A very interesting class of genes, involved in selenium metabolism is under investigation with respect to different kind of cancers including breast cancer. Proteins coded by these genes, e.g. glutathione peroxidases, thioredoxin reductases or other selenoproteins are involved in variety of biological processes, ranging from DNA synthesis to protection against oxidative stress and may be related to breast cancer risk.

Aim of our study was to analyze methylation of genes involved in selenium metabolism (GPX1, GPX4, TXNRD1, SEP15 and SELT) and other cancer related genes (BRCA1, $B R C A 2, A T M, M L H 1$ and ESR1) in DNA isolated from PB of breast cancer patients or unaffected individuals.

A study was conducted on 30 female BRCA1 mutation carriers, 30 female CHEK2 mutation carriers and 36 unselected breast cancer patients negative for recurrent Polish BRCA1/BRCA2 mutations and with specific pathological characteristics: medullary or atypical medullary breast cancer or bilateral breast cancer or ER, PGR and HER negative tumors. Methylation of BRCA1 gene was additionally analyzed in group of 36 healthy controls, age-matched to unselected breast cancer patients. Methylation analysis was done by MS-HRM technique.

We observed promoter methylation of BRCA1 (20 samples), ESR1 (7 samples) and MLH1 (3 samples) genes. $B R C A 1$ gene methylation was detected in 1 patient with CHEK2 and 4 patients with BRCA1 mutation. We found strong statistically significant difference of BRCA1 gene methylation in unselected breast cancer patients (15 patients) and unaffected age-matched controls (2 individuals): $O R=12.1 ; p$-value $=0.0009$. Selenoprotein coding genes GPX1 and GPX4 shows complete methylation while TXNRD1 and SELT show methylation in $\sim 10 \%$ of the studied group.

\section{Conclusion}

Constitutional methylation (in peripheral blood) of BRCA1 gene seems to be a strong factor risk of breast cancer with specific pathological characteristic.

\section{Author details}

'Department of Genetics and Pathology, Pomeranian Medical University, Szczecin, Poland. ${ }^{2}$ Institute of Human Genetics, Aarhus University, Aarhus, Denmark. ${ }^{3}$ Postgraduate School of Molecular Medicine, Warsaw Medical University, Poland.

Published: 10 December 2012

doi:10.1186/1897-4287-10-S4-A13

Cite this article as: Gupta et al:: Constitutional methylation of cancerrelated and selenoprotein coding genes in breast carcinoma in Polish population. Hereditary Cancer in Clinical Practice 2012 10(Suppl 4):A13. 